To appear in the Journal of Control and Decision

Vol. 00, No. 00, Month 20XX, 1-26

\title{
Distributed Schemes for Efficient Deployment of Price-Responsive Demand with Partial Flexibility
}

\author{
A. De Paola ${ }^{\dagger * a}$, D. Angeli ${ }^{\dagger b}$ and G. Strbac ${ }^{\dagger c}$ \\ ${ }^{\dagger}$ Department of Electrical and Electronic Engineering, Imperial College London, \\ South Kensington Campus, SW7 2AZ, London, UK
}

(v1.2 submitted July 2017)

This work was supported by the EPSRC under Grant [EP/I031650/1] and Grant [EP/K002252/1].

${ }^{*}$ Corresponding author.

${ }^{a}$ Email: antonio.de-paola09@imperial.ac.uk. Tel.: +44(0)7423194121. ORCID: 0000-0003-0312-2567.

${ }^{b}$ Email: d.angeli@imperial.ac.uk. Tel.: +44 (0)2075946283. ORCID: 0000-0002-5311-4306.

${ }^{c}$ Email: g.strbac@imperial.ac.uk. Tel.: +44 (0)2075946169. 


\begin{abstract}
This paper presents novel methodologies for efficient deployment of flexible demand. Large populations of price-responsive loads are coordinated through a price signal and a power constraint broadcast by a central entity. Such quantities are designed in order to minimise a global objective function (e.g. total generation costs) and ensure a one-step convergence to a stable solution, characterised as a Nash equilibrium. Conditions for the sought equilibrium are preliminarily expressed as monotonicity of demand profiles under reordered coordinates and then they are imposed as constraints of a global optimisation, whose solution is calculated numerically. To reduce the computational complexity of the problem in scenarios with high penetration of flexible demand, clustering of the appliances is introduced. The global properties of the final stable solution and its optimality with respect to the task times of the appliances are analysed both theoretically and through simulation results.
\end{abstract}

Keywords: electric power network; demand response; game theory; distributed control;

\title{
1. Introduction
}

A defining element of the transition of power systems towards the smart grid paradigm is the increasing penetration of flexible demand. New typologies of loads, such as 'smart' appliances and electric vehicles, are seeing a growing diffusion. These devices are able to schedule their power consumption with a certain flexibility, on the basis of customers requirements and energy price during the day. The possible advantages deriving by the diffusion of these new technologies have been analysed in detail (Albadi \& El-Saadany, 2008; Strbac, 2008): electricity bills will be cheaper for private customers and the power system will benefit from an improved reliability and reduced reliance on fossil fuel peaking plants. In order to exploit the full potential of flexible demand, it is necessary to devise a proper coordination of the appliances, achieving a good trade-off between the requirements of the individual consumers and the global objectives of the power system. Centralised solutions have been proposed to efficiently solve this problem (Samadi, Mohsenian-Rad, Schober, \& Wong, 2012; Su \& Kirschen, 2009). These approaches envisage a central entity that clears the market by solving a global optimisation, on the basis of the data broadcast by generators and consumers. Such strategies can require considerable computational resources and communication infrastructure if large power networks are considered. For this reason, a wide range of distributed techniques have also been proposed. In (Z. Chen, Wu, \& Fu, 2012) the coordination of the flexible devices is achieved through a stochastic optimisation while (Fan, 2012) presents an adaptive strategy based on price feedbacks and (Mohsenian-Rad, Wong, Jatskevich, Schober, \& Leon-Garcia, 2010) applies game theory, determining the energy consumption of the flexible devices as the best response to certain energy price signals. At high penetration levels of flexible demand, the main problem faced by these decentralised techniques is the synchronisation of the devices, as all flexible loads will tend to operate when electricity is cheaper. Their operation strategies will increase aggregate power demand and electricity prices at those times, resulting in suboptimality of their original power scheduling. Different schemes have been devised to avoid synchronisation: (Ruthe, Rehtanz, \& Lehnhoff, 2015) proposes to broadcast a randomised price to each appliance whereas (Boait, Ardestani, \& Snape, 2013) introduces randomness on the controllers of the individual devices, considering an intermediate entity (aggregator) between the energy market and the individual customer. This paper follows an approach similar to (Papadaskalopoulos \& Strbac, 2013), which introduces a proportional constraint on the power rate of the devices in order to saturate flexible demand at certain time instants and avoid rebound peaks. The mentioned work considers such constraint as constant over time and selects it heuristically, iteratively modifying the imposed power saturation until the less stringent value that ensures a feasible solution is obtained. In the present paper, the proportional power constraint is 
chosen as a function of time (allowing a smoother allocation of flexible demand) and it is analytically calculated so as to optimise a global performance index while ensuring a stable market configuration.

The distributed control strategy proposed in this work is formulated within a game theory framework. The appliances are modelled as competitive players that interact through the changes in energy price introduced by their scheduled power consumption. On the basis of a price profile and a proportional power constraint broadcast by a central entity, the appliances schedule their power consumption in order to complete their task at minimum energy cost. The broadcast signals are calculated in order to optimise some global index that can quantify, for example, the generation costs or the flattening of aggregate demand. In addition, the obtained solution corresponds to a Nash equilibrium: the individual agent has no interest in modifying its scheduled power consumption (formulated on the basis of the initially broadcast signals) when the final energy price is considered. Similar results have been achieved by adding additional terms in the cost function of the appliances (Gan, Topcu, \& Low, 2013; Ma, Callaway, \& Hiskens, 2013) or under some condition on the electricity price and the number of devices (H. Chen, Li, Louie, \& Vucetic, 2014; Ma, Zou, Ran, Shi, \& Hiskens, 2016). In these works, the equilibrium is reached in a distributed manner by iterated broadcasts of price/demand signals to the flexible appliances, which in turn update their power scheduling in order to minimise their expected energy costs. This scheme is quite common in problems of flexible demand coordination and, for example, it is also adopted by (Mohsenian-Rad et al., 2010), albeit with a different pricing structure and asynchronous random updates. The novelty of our approach is that it does not require repeated exchange of information between the system operator and the appliances, obtaining convergence to equilibrium through the broadcast of a single price signal (together with a proportional power constraint). Moreover, the cost function of the appliances corresponds to their actual energy payments and only the monotonicity of the electricity price function needs to be assumed. To achieve these results, the proposed approach explicitly characterises the equilibrium as inequality constraints in the global optimisation problem. Using the analysis in (De Paola, Angeli, \& Strbac, 2015) as a starting point, this is obtained by imposing monotonicity of the aggregate demand profile, evaluated over a reordered time vector. The solution of the constrained optimisation problem is first calculated numerically and then characterised by a backward integration of a dynamical system, adopting the approach initially presented in (De Paola, Angeli, $\&$ Strbac, 2016). The optimisation is performed in one step and does not require precise knowledge of the electricity price function. The main difference with respect to the cited works is that the analysis now includes optimisation of system objectives and is conducted on a finite number of appliances. Moreover, partial flexibility is taken into account: each agent can specify a subset of the considered time horizon during which it is available to operate. Clustering techniques are also discussed, in order to guarantee computational scalability when the number of flexible loads is significantly high.

The rest of the paper is structured as follows: Section 2 presents the modelling choices for the power system and introduces the distributed control scheme. The Nash equilibrium conditions and the design of the control signals are detailed in Section 3. The clustering technique used to reduce the computational complexity of the problem is described in Section 4 while simulation results are presented in Section 5. Finally, analytical characterisation of the proposed solution and its properties with respect to the appliances task time are analysed in Section 6. 


\section{Modelling Framework and Distributed Coordination}

As a preliminary step, the considered system components and their interactions (summarised in Fig. 1) are modelled.

\subsection{Flexible Appliances and Electricity Market}

We consider a population $\mathscr{N}=\{1, \ldots, N\}$ of flexible appliances, operating over the discrete time interval $\mathscr{T}=\{1, \ldots, T\}$. Each device $j \in \mathscr{N}$ is characterised by three quantities: its rated power $P_{j}^{\mathrm{r}}$, the required energy $E_{j}^{\mathrm{r}}$ for task completion (or alternatively the minimum task time at rated power $t_{j}^{\mathrm{r}}=E_{j}^{\mathrm{r}} / P_{j}^{\mathrm{r}}$ ) and the set of time instants $\mathscr{A}_{j} \subseteq \mathscr{T}$ during which it is available to operate. The power consumption of the $j$-th device over the considered time horizon $\mathscr{T}$ is denoted as $u_{j}: \mathscr{T} \rightarrow\left[0, P_{j}^{\mathrm{r}}\right]$.

The electricity market has been abstracted by the price function $\Pi: \mathrm{R}_{+} \rightarrow \mathrm{R}_{+}$which associates, to a certain power demand $D$, the corresponding electricity price $\Pi(D)$. The final electricity price at each time instant $t \in \mathscr{T}$ is given by $\Pi\left(D_{a}(t)\right)$, where $D_{a}(t)$ corresponds to the aggregate power demand in the system. This quantity is given by the sum of two distinct terms: the power consumption $D_{i}(t)$ of the inflexible loads (assumed to be known a priori) and the total contribution $D_{f}(t)$ of the flexible appliances:

$$
D_{a}(t)=D_{i}(t)+D_{f}(t)=D_{i}(t)+\sum_{j=1}^{N} u_{j}(t)
$$

Assumption 1 The price function $\Pi(D)$ is strictly monotone increasing.

As a first approximation, one can reasonably assume that electricity will be more expensive when more power needs to be generated to meet higher demand. Note that precise knowledge of the price function $\Pi$ is not required, as long as its monotonicity property is satisfied.

Assumption 2 The single device $j$ has no market power and cannot significantly impact prices through its power consumption:

$$
\Pi\left(D_{a}(t)\right)=\Pi\left(D_{i}(t)+\sum_{k=1}^{N} u_{k}(t)\right) \simeq \Pi\left(D_{i}(t)+\sum_{k \in \mathscr{N} \backslash j} u_{k}(t)\right) .
$$

This assumption realistically holds if large numbers of small appliances (with maximum power consumption $P^{\mathrm{r}}$ in the order of kilowatts) are considered in the context of large power systems (where total demand is in the order of gigawatts).

\subsection{Distributed Coordination Scheme}

In order to efficiently integrate flexible demand in the power system, a distributed control strategy is considered. As a preliminary step, the devices communicate their parameters $\left(P_{j}^{\mathrm{r}}, E_{j}^{\mathrm{r}}\right.$ and $\left.\mathscr{A}_{j}^{\mathrm{r}}\right)$ to the system operator. This, in turn, broadcasts a price function $p$ : $\mathscr{T} \rightarrow \mathrm{R}_{+}$and a signal $\alpha: \mathscr{T} \rightarrow[0,1]$. The latter represents a time-varying proportional constraint (equal for all devices) on power consumption.

Remark 1 The purpose of the power constraint $\alpha$ is to saturate flexible demand at critical time instants and achieve an equilibrium, as detailed in subsequent sections. It can be 
directly enforced on the appliances or implemented through discontinuous prices, consistently penalizing the devices that exceed their prescribed fraction of power consumption.

The appliances are supposed to be price-responsive and schedule their power consumption in order to complete their task at minimum cost. The power profile $u_{j}^{*}(\cdot, p, \alpha): \mathscr{T} \rightarrow$ $\left[0, P_{j}^{\mathrm{r}}\right]$ scheduled by the $j$-th device when $p$ and $\alpha$ are broadcast, fulfils the following condition:

$$
\begin{aligned}
& u_{j}^{*}(\cdot, p, \alpha) \in \arg \min _{u(\cdot)} \sum_{t=1}^{T} p(t) u(t) \Delta t \\
& \text { s. t. } \sum_{t=1}^{T} u(t) \Delta t=E_{j}^{\mathrm{r}} \\
& \quad 0 \leq u(t) \leq \alpha(t) P_{j}^{\mathrm{r}} \cdot \mathbb{1}_{\mathscr{A}_{j}}(t) \quad \forall t \in\{1, \ldots, T\}
\end{aligned}
$$

where $\Delta t$ is the time discretisation step and $\mathbb{1}_{\mathscr{S}}(\cdot)$ denotes the indicator function. The objective function in (2a) corresponds to the expected cost sustained by the $j$-th device. It is equal to the sum over the time index $t$ of the electricity price $p(t)$, multiplied by the consumed energy $u(t) \Delta t$. The constraint ( $2 \mathrm{~b}$ ) ensures task completion while ( $2 \mathrm{c}$ ) guarantees that, at each time $t$, the consumed power is within feasible bounds. In particular, if the $j$-th device is available at time $t\left(t \in \mathscr{A}_{j}\right.$ and $\left.\mathbb{1}_{\mathscr{A}_{j}}(t)=1\right)$, its power cannot be greater than $\alpha(t) P_{j}^{\mathrm{r}}$. In the opposite case, with $\mathbb{1}_{\mathscr{A}_{j}}(t)=0$, power cannot be consumed.

Lemma 1 The optimisation problem in (2) is feasible for a certain $\alpha(\cdot)$ if and only if the $j$-th device can complete its task in the specified availability interval by operating at the maximum allowed power:

$$
\sum_{t=1}^{T}\left(\alpha(t) P_{j}^{r} \cdot \mathbb{1}_{\mathscr{A}_{j}}(t)\right) \Delta t=\sum_{t \in \mathscr{A}_{j}}\left(\alpha(t) P_{j}^{r}\right) \Delta t \geq E_{j}^{r}
$$

\subsection{Power Scheduling in Reordered Time Coordinates}

In the proposed distributed scheme, appliances coordination is achieved by broadcasting the signals $p$ and $\alpha$ and letting each device $j$ independently determine its cost-minimising power scheduling $u_{j}^{*}(\cdot, p, \alpha)$. To simplify the subsequent analysis and obtain compact expressions for $u_{j}^{*}$, the time vector $\mathscr{T}$ is reordered according to increasing prices.

Definition 1 Consider an injective price function $p:[0, T] \rightarrow \mathrm{R}_{+}$and the associated $f_{p}: \mathscr{T} \rightarrow \mathscr{T}$, fulfilling the following condition:

$$
p\left(f_{p}(1)\right)<p\left(f_{p}(2)\right)<\cdots<p\left(f_{p}(T)\right) .
$$

For any signal $g: \mathscr{T} \rightarrow \mathrm{R}$ it is possible to define the corresponding $\bar{g}$ (denoted with a bar superscript) evaluated over the reordered time vector $f_{p}(t)$ :

$$
\bar{g}(t):=g\left(f_{p}(t)\right) \quad \forall t \in \mathscr{T} .
$$

The same notation with bar superscript can be extended to any time subset $\mathscr{I}=\left\{t_{1}, \ldots, t_{n}\right\} \subseteq \mathscr{T}$, to which corresponds $\overline{\mathscr{I}}=\left\{f_{p}^{-1}\left(t_{1}\right), \ldots, f_{p}^{-1}\left(t_{n}\right)\right\}$. 
Remark 2 In the rest of the paper, the price function $p$ is always chosen to be injective $\left(p\left(t_{1}\right) \neq p\left(t_{2}\right) \forall t_{1}, t_{2} \in \mathscr{T}\right)$ so as to allow the transformation detailed above.

For a qualitative representation of the considered time reordering, some examples are presented in Fig. 2. The time reordering is performed according to the price signal $p$ presented in the top-left. As expected, the resulting $\bar{p}$ is monotone increasing in the new time coordinates. Note that such monotonicity properties do not hold in general for an arbitrary function $g$, as shown in the right side of the figure.

The scheduling problem of the individual device can now be considered in the reordered time vector, characterised by increasing price values:

$$
\begin{aligned}
\min _{\bar{u}(\cdot)} & \sum_{t=1}^{T} \bar{p}(t) \bar{u}(t) \Delta t \\
\text { s. t. } & \sum_{t=1}^{T} \bar{u}(t) \Delta t=E_{j}^{\mathrm{r}} \\
& 0 \leq \bar{u}(t) \leq \bar{\alpha}(t) P_{j}^{\mathrm{r}} \cdot \mathbb{1}_{\overline{\mathscr{A}}_{j}}(t) \quad \forall t \in\{1, \ldots, T\}
\end{aligned}
$$

where $\bar{p}, \bar{\alpha}$ and $\overline{\mathscr{A}}_{j}$ represent the equivalent of $p, \alpha$ and $\mathscr{A}_{j}$, as specified in Definition 1 . By exploiting the particular structure of the cost function in (6) and the monotonicity of the broadcast price $\bar{p}$, it is possible to provide a closed-form expression for the corresponding minimiser $\bar{u}_{j}^{*}$.

Proposition 1 For any signal $\alpha(\cdot)$ fulfilling (3), the optimisation problem (6) has a unique minimiser $\bar{u}_{j}^{*}(\cdot, \bar{\alpha})$, with the following expression:

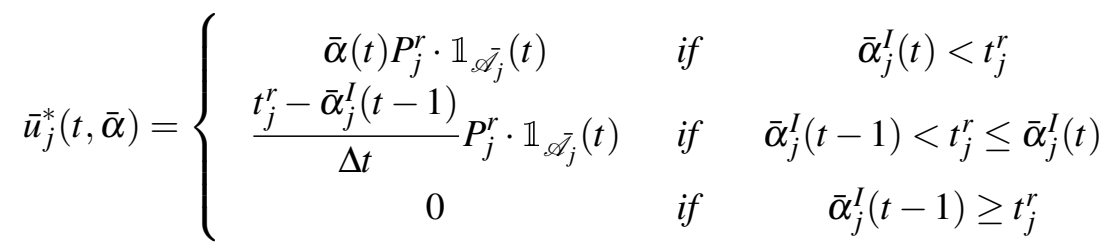

where $t_{j}^{r}=E_{j}^{r} / P_{j}^{r}$ and the quantity $\bar{\alpha}_{j}^{I}(t)$ is defined as:

$$
\bar{\alpha}_{j}^{I}(t):=\sum_{l \in\left(\mathscr{\alpha}_{j} \cap\{1, \ldots, t\}\right)} \bar{\alpha}(l) \cdot \Delta t .
$$

Proof. See Appendix A.

Since $\bar{\alpha}_{j}^{I}$ is monotone increasing (sum of positive terms over an interval of increasing size), each device will operate at maximum feasible power during the first reordered time instants (until $\bar{\alpha}_{j}^{I}(t)<t_{j}^{\mathrm{r}}$ ). This interval corresponds to the lowest values of the electricity price $\bar{p}$, given its strict monotonicity in the reordered coordinates. The only exception is represented by the second case in (7), i.e. the last instant of operation of the $j$-th device. At this specific time, the remaining required power may be lower than the maximum feasible one and therefore $\bar{u}_{j}^{*}$ must be adjusted accordingly.

Note that the dependency of $\bar{u}^{*}$ on the price signal $p$ is implicit through the reordered coordinates and therefore does not appear explicitly in the notation. Moreover, to obtain the power scheduling $u^{*}$ and the power constraint $\alpha$ in the original time variable, it is sufficient to perform the inverse of the operation detailed in Definition 1:

$$
u_{j}^{*}(t, p, \alpha)=\bar{u}_{j}^{*}\left(f_{p}^{-1}(t), \bar{\alpha}\right) \quad \alpha(t)=\bar{\alpha}\left(f_{p}^{-1}(t)\right) .
$$




\section{Control Signals Design}

We are interested in calculating a price signal $p$ and a constraint function $\alpha$ that, when broadcast to the flexible appliances, induce a suitable allocation of their aggregate power consumption. This design objective is characterised as a Nash equilibrium: when the final energy price $\Pi\left(D_{a}\right)$ is considered, no individual device can achieve a reduced energy cost by unilaterally changing its initially scheduled power. This condition has been commonly adopted by similar works on distributed coordination of flexible demand, such as (Ma et al., 2013) and (Mohsenian-Rad et al., 2010), since it corresponds to a 'fair' solution for all the flexible agents. Moreover, from a global point of view, synchronicity phenomena are avoided, achieving a flattened aggregate demand profile and reduced generation costs.

\subsection{Conditions for Nash Equilibrium}

The equilibrium concept is applied to a static game with the following components:

- Players: The population $\mathscr{N}=\{1, \ldots, N\}$ of flexible appliances.

- Strategies: Feasible power profiles $u_{j}: \mathscr{T} \rightarrow\left[0, P_{j}^{\mathrm{r}}\right]$ that guarantee task completion, i.e. fulfil ( $2 \mathrm{~b})$ and (2c). The $j$-th device schedules $u_{j}=u_{j}^{*}$ by solving the optimisation problem (2), on the basis of the broadcast signals $p$ and $\alpha$.

- Objective function: Minimisation of the total energy $\operatorname{cost} C_{j}$ sustained by the single device $j$ to complete its task:

$$
C_{j}=\sum_{t=1}^{T} \Pi\left(D_{a}(t)\right) u_{j}(t) \Delta t
$$

The notion of Nash equilibrium for this game can be defined as follows:

Definition 2 Given the broadcast signals $p$ and $\alpha$, the resulting scheduled power profiles $u_{j}(\cdot)=u_{j}^{*}(\cdot, p, \alpha)$ correspond to a Nash equilibrium if the following holds for all $j \in \mathscr{N}$ :

$$
\begin{aligned}
\sum_{t=1}^{T} \Pi\left(D_{a}^{*}(t, p, \alpha)\right) u_{j}^{*}(t, p, \alpha) \Delta t= & \min _{u(\cdot)} \sum_{t=1}^{T} \Pi\left(D_{a}^{*}(t, p, \alpha)\right) u(t) \Delta t \\
& \text { s. t. } \sum_{t=1}^{T} u(t) \Delta t=E_{j}^{\mathrm{r}} \\
& 0 \leq u(t) \leq \alpha(t) P_{j}^{\mathrm{r}} \cdot \mathbb{1}_{\mathscr{A}_{j}}(t) \quad \forall t \in\{1, \ldots, T\} .
\end{aligned}
$$

where the aggregate demand profile $D_{a}^{*}$ is defined as:

$$
D_{a}^{*}(t, p, \alpha)=D_{i}(t)+\sum_{j=1}^{N} u_{j}^{*}(t, p, \alpha)
$$

The proposed definition is conceptually similar to the equilibrium notions recently presented in (Lasry \& Lions, 2007) and (Olivier, Lasry, \& Lions, 2011) for games with infinite players, or mean field games. In these works it is assumed that the impact of the single (infinitesimal) player on the global quantities of the system is negligible and therefore only the mass behaviour of the players' population (mean field) needs to be considered. A similar situation arises in the present case: it has been established in Assumption 2 that the impact of the single device on the electricity price is negligible and therefore only the total power consumption $D_{f}(t)=\sum_{i=1}^{N} u_{j}(t)$ of the flexible appliances needs to be taken 
into account. It follows that, at equilibrium, each power profile $u_{j}^{*}$ is cost-minimising for a certain aggregate demand $D_{a}^{*}$ (through (10)) and, at the same time, the whole set of power schedules induces that very same demand profile, as imposed by (11). Similar equilibrium concepts have been adopted by other works analyzing the coordination of large populations of flexible appliances, such as (Ma et al., 2013) and (Grammatico, Parise, Colombino, \& Lygeros, 2016).

As a preliminary step in the design of the power constraint $\alpha$, an equivalent equilibrium condition is expressed by means of inequalities on the aggregate demand:

Proposition 2 Denote by $\bar{D}_{a}^{*}$ the aggregate demand evaluated over the reordered time vector $f_{p}$ (as specified in Definition 1):

$$
\bar{D}_{a}^{*}(t, \bar{\alpha}):=D_{a}^{*}\left(f_{p}(t), p, \alpha\right)
$$

A Nash equilibrium is achieved according to Definition 2 and (10)-(11) are fulfilled if:

$$
\bar{D}_{a}^{*}(1, \bar{\alpha}) \leq \bar{D}_{a}^{*}(2, \bar{\alpha}) \leq \cdots \leq \bar{D}_{a}^{*}(T, \bar{\alpha})
$$

Proof. It is straightforward to derive the equivalent of the optimisation problem in (10) when the reordered time vector is considered:

$$
\begin{aligned}
& \min _{\bar{u}(\cdot)} \sum_{t=1}^{T} \Pi\left(\bar{D}_{a}^{*}(t, \bar{\alpha})\right) \bar{u}(t) \Delta t \\
& \text { s. t. } \sum_{t=1}^{T} \bar{u}(t) \Delta t=E_{j}^{\mathrm{r}} \\
& \quad 0 \leq \bar{u}(t) \leq \bar{\alpha}(t) P_{j}^{\mathrm{r}} \cdot \mathbb{1}_{\mathscr{A}_{j}}(t) \quad \forall t \in\{1, \ldots, T\} .
\end{aligned}
$$

It can be shown that $\bar{u}_{j}^{*}(\cdot, \bar{\alpha})$ is optimal for (14) using the same arguments of the proof of Proposition 1. Any $\bar{u}_{j}$ fulfilling the constraints in (14) can be written as $\bar{u}_{j}=\bar{u}_{j}^{*}+\sum_{l=1}^{L} \delta_{l}$, i.e. as the sum of $\bar{u}_{j}^{*}$ and $L$ finite variations $\delta_{l}$. The terms $\delta_{l}$ are defined as in (A2), with $\varepsilon>0$ and the parameters $x_{1}$ and $x_{2}$ fulfilling (A3). If (13) holds, since the price function $\Pi$ is strictly monotone increasing and $x_{2} \geq x_{1}$, we have:

$$
\sum_{t=1}^{T} \Pi\left(\bar{D}_{a}^{*}(t, \bar{\alpha})\right) \delta_{l}(t) \Delta t=\varepsilon\left[\Pi\left(\bar{D}_{a}\left(x_{2}, \bar{\alpha}\right)\right)-\Pi\left(\bar{D}_{a}\left(x_{1}, \bar{\alpha}\right)\right)\right] \Delta t \geq 0 \quad l=1, \ldots, L
$$

The optimality of $\bar{u}_{j}^{*}(\cdot, \bar{\alpha})$ for (14) follows from the linearity of the cost function in (14) with respect to $\bar{u}_{j}=\bar{u}_{j}^{*}+\sum_{l=1}^{L} \delta_{l}$. As a result, the corresponding $u_{j}^{*}(\cdot, p, \alpha)$ in the original time variable is optimal for the minimisation problem in (10).

From Proposition 2, a Nash equilibrium is successfully induced by the broadcast signals $p$ and $\alpha$ if the resulting aggregate demand $\bar{D}_{a}^{*}(\cdot, \bar{\alpha})$, evaluated over the reordered time coordinates determined by $p$, is nondecreasing.

\subsection{Global Optimisation Problem}

The broadcast price $p$ and constraint $\alpha$ are designed in order to optimise a global index of the resulting aggregate demand $D_{a}^{*}(t, p, \alpha)$, defined in (11). The chosen functional $J$ to be 
minimised has the following expression:

$$
J\left(D_{a}^{*}(\cdot)\right)=\sum_{t=1}^{T} U\left(D_{a}^{*}(t, p, \alpha)\right)
$$

where $U$ is a convex positive function. This choice can quantify, for example, the flattening of the aggregate demand profile introduced by the flexible appliances or the total generation costs of the system. Note that, if the functional is considered in the reordered time variable, the inequalities in (13) can be imposed as optimisation constraints, guaranteeing that the optimal solution is also a Nash equilibrium.

Remark 3 To simplify the analysis, the broadcast signal $p$ is chosen as the price of inflexible demand, with $p(t)=\Pi\left(D_{i}(t)\right)$.

This price selection is optimal when all devices have total flexibility and corresponds to a 'rational' scheduling since the flexible agents will allocate more power when the base profile $D_{i}$ is lower. The possibility of broadcasting different price signals, for example taking into account the availability of the appliances at different times, will be considered in a future work.

If one operates with the reordered time vector introduced in Definition 1, the aggregate demand (12) and the global cost functional (15) become:

$$
\bar{D}_{a}^{*}(t, \bar{\alpha})=\bar{D}_{i}(t)+\sum_{j=1}^{N} \bar{u}_{j}^{*}(t, \bar{\alpha}) \quad J\left(D_{a}^{*}(\cdot)\right)=\sum_{t=1}^{T} U\left(\bar{D}_{a}^{*}(t, \bar{\alpha})\right) .
$$

The optimal power constraint $\bar{\alpha}$ which induces a Nash equilibrium can be calculated through the following optimisation problem:

$$
\begin{aligned}
& \min _{\bar{\alpha}(\cdot)} \sum_{t=1}^{T} U\left(\bar{D}_{a}^{*}(t, \bar{\alpha})\right) \\
& \text { s.t. } 0 \leq \bar{\alpha}(t) \leq 1 \\
& \forall t \in \mathscr{T} \\
& \bar{D}_{a}^{*}(t, \bar{\alpha}) \leq \bar{D}_{a}^{*}(t+1, \bar{\alpha}) \\
& \forall t \in\{1, \ldots, T-1\} \\
& \sum_{t \in \bar{A}_{j}} \bar{\alpha}(t) \geq t_{j}^{\mathrm{r}} \\
& \forall j \in \mathscr{N} .
\end{aligned}
$$

The constraint (17b) corresponds to the choice of a proportional limitation on the power rate while (17c) is equal to the equilibrium condition (13). The inequalities in (17d) are equivalent to (3) in Lemma 1 and ensure that the chosen $\bar{\alpha}$ allows all appliances to complete their tasks within their availability window $\overline{\mathscr{A}}_{j}$. Once the optimal $\bar{\alpha}^{*}$ has been calculated for the minimisation problem (17), it is straightforward to obtain the equivalent $\alpha^{*}$ in the original time variable by applying (9).

Remark 4 Albeit not formally proven for length reasons, it can be shown that the proposed distributed scheme is 'fair': loads with equal parameters will complete their tasks at equal prices. Moreover, flexibility is incentivised as lower costs will be sustained when larger availability-time windows are declared. An example of this property for the simulated case study is provided in Fig. 4. 


\section{Clustering of the Devices}

For large populations of flexible appliances, it is in general difficult to show existence and provide a closed-form expression for the solution of (17). For this reason, the optimisation has been solved with numerical methods. In this respect, it is worth noticing that problem (17) presents $T$ decision variables and $2 T+(T-1)+N$ inequality constraints. While the number of time instants $T=|\mathscr{T}|$ is relatively low, the number of devices $N=|\mathscr{N}|$ could be in the order of millions. Therefore, when large populations of devices are considered, it is in general difficult to calculate the numerical solution in reasonable time. To reduce the computational complexity of the constrained optimisation, a clustering of the appliances is introduced, representing devices with similar properties as a unique macro-player. Some assumptions are preliminarily made on the availability of the devices:

Assumption 3 The set $\mathscr{A}_{j}$, characterising the time availability of the $j$-th appliance, always corresponds to a unique interval:

$$
\mathscr{A}_{j}=\left\{t_{j}^{\text {in }}, t_{j}^{\text {in }}+1, \ldots, t_{j}^{\text {fin }}-1, t_{j}^{\text {fin }}\right\} \subseteq \mathscr{T}
$$

This means that the $j$-th device can be unequivocally characterised by only four parameters: rated power $P_{j}^{\mathrm{r}}$, rated task time $t_{j}^{\mathrm{r}}=E_{j}^{\mathrm{r}} / P_{j}^{\mathrm{r}}$ and initial/final availability time (respectively $t_{j}^{\mathrm{in}}$ and $t_{j}^{\mathrm{fin}}$ ).

Suppose now that the time parameters are equal for a certain set $\mathscr{L} \subseteq \mathscr{N}$ of devices:

$$
t_{j}^{\mathrm{r}}=\hat{t}^{\mathrm{r}} \quad t_{j}^{\text {in }}=\hat{t}^{\text {in }} \quad t_{j}^{\mathrm{fin}}=\hat{t}^{\mathrm{fin}} \quad \forall j \in \mathscr{L}
$$

In this case, an optimisation problem equivalent to (17) can be introduced by replacing the set $\mathscr{L}$ (and the corresponding constraints) with a single device $\hat{j}$ that has the following characteristics:

$$
t_{\hat{j}}^{\mathrm{r}}=\hat{t}^{\mathrm{r}} \quad t_{\hat{j}}^{\mathrm{in}}=\hat{t}^{\mathrm{in}} \quad t_{\hat{j}}^{\mathrm{fin}}=\hat{t}^{\mathrm{fin}} \quad P_{\hat{j}}^{\mathrm{r}}=\sum_{l \in \mathscr{L}} P_{l}^{\mathrm{r}} .
$$

In defining the clustering of the appliances, we assume that the rated task time $t^{\mathrm{r}}$ of each macro player can only take values in the following finite set:

$$
\mathscr{T}^{\mathrm{r}}=\left\{\frac{1}{k}, \frac{2}{k}, \ldots, \frac{T k}{k}\right\} .
$$

The quantity $k$ represents a design parameter that can be tuned to achieve the desired tradeoff between accuracy of the clustering approximation and computational time required to solve the optimisation problem. The individual appliances can then be grouped in a finite number of sets $\hat{N}_{x}^{y, z}$ such that:

$$
t_{j}^{\mathrm{r}} \in\left(\frac{x-1}{k}, \frac{x}{k}\right] \quad t_{j}^{\mathrm{in}}=y \quad t_{j}^{\mathrm{fin}}=z \quad \forall j \in \hat{\mathscr{N}}_{x}^{y, z} .
$$

It is possible to define a set $\hat{\mathscr{N}}$ of aggregated appliances (with cardinality $\hat{N}$ ) where each element $\hat{j}$ is associated to a different set of appliances $\hat{\mathscr{N}}_{x}^{y, z} \subseteq \mathscr{N}$ and has the following parameters:

$$
t_{\hat{j}}^{\mathrm{r}}=\frac{x}{k} \quad t_{\hat{j}}^{\mathrm{in}}=y \quad t_{\hat{j}}^{\mathrm{fin}}=z \quad P_{\hat{j}}^{\mathrm{r}}=\sum_{l \in \hat{\mathscr{Y}} \hat{x}, z} P_{l}^{\mathrm{r}} .
$$


The total power consumption of the devices in a certain set $\hat{\mathscr{N}}_{x}^{y, z}$ can be approximated as the best response $\bar{u}_{\hat{j}}$ of the corresponding macro-appliance $\hat{j}$, characterised as follows:

$$
\begin{aligned}
\bar{u}_{\hat{j}}^{\diamond}(\cdot, \bar{\alpha}) \in & \arg \min _{\bar{u}(\cdot)} \sum_{t=1}^{T} \bar{p}(t) \bar{u}(t) \Delta t \\
\text { s. t. } \quad & \sum_{t=1}^{T} \bar{u}(t) \Delta t=P_{\hat{j}}^{\mathrm{r}} \cdot t_{\hat{j}}^{\mathrm{r}}=E_{\hat{j}}^{\mathrm{r}} \\
& 0 \leq \bar{u}(t) \leq \bar{\alpha}(t) P_{\hat{j}}^{\mathrm{r}} \cdot \mathbb{1}_{\mathscr{A}_{\hat{j}}}(t) \quad \forall t \in\{1, \ldots, T\} .
\end{aligned}
$$

One can verify that its expression is equal to (7) if one considers $P_{\hat{j}}^{\mathrm{r}}, E_{\hat{j}}^{\mathrm{r}}$ and $\overline{\mathscr{A}} \overline{\hat{j}}$ as appliances parameters, where $\overline{\mathscr{A}}_{\hat{j}}$ has the following expression:

$$
\overline{\mathscr{A}}_{\hat{j}}=\left\{t: f_{p}(t) \geq t_{\hat{j}}^{\mathrm{in}}, f_{p}(t) \leq t_{\hat{j}}^{\mathrm{fin}}\right\} .
$$

Similarly, the aggregate demand $D_{a}^{*}$ can be approximated as $D_{a}^{\diamond}$, considering the total power consumption $D_{f}^{\diamond}$ of the macro-appliances as the contribution of flexible demand:

$$
\bar{D}_{f}^{\diamond}(t)=\sum_{j=1}^{\hat{N}} \bar{u}_{j}^{\diamond}(t, \bar{\alpha}) \quad \bar{D}_{a}^{\diamond}(t, \bar{\alpha})=\bar{D}_{i}(t)+\bar{D}_{f}^{\diamond}(t)=\bar{D}_{i}(t)+\sum_{j=1}^{\hat{N}} \bar{u}_{j}^{\diamond}(t, \bar{\alpha}) .
$$

An approximation of the global optimisation problem (17) can now be solved considering the clustered appliances:

$$
\begin{aligned}
& \min _{\bar{\alpha}(\cdot)} \sum_{t=1}^{T} U\left(\bar{D}_{a}^{\diamond}(t, \bar{\alpha})\right) \\
& \text { s.t. } 0 \leq \bar{\alpha}(t) \leq 1 \\
& \forall t \in \mathscr{T} \\
& \bar{D}_{a}^{\diamond}(t, \bar{\alpha}) \leq \bar{D}_{a}^{\diamond}(t+1, \bar{\alpha}) \\
& \forall t \in\{1, \ldots, T-1\} \\
& \sum_{t \in \mathscr{A} \overline{\hat{j}}} \bar{\alpha}(t) \geq t_{\hat{j}}^{\mathrm{r}} \\
& \forall \hat{j} \in \hat{\mathscr{N}} \text {. }
\end{aligned}
$$

Note that (27) presents the same structure of problem (17), the only difference is that the set $\hat{\mathscr{N}}$ of macro-appliances is smaller than $\mathscr{N}$ and therefore a reduced number of constraints must be considered.

Remark 5 It is possible to determine an upper bound on the number of aggregated appliances $\hat{N}=|\hat{N}|$ obtained when the clustering described above is applied. In fact, for each $\hat{j} \in \hat{\mathscr{N}}$, it must hold:

$$
1 \leq t_{\hat{j}}^{\text {in }} \leq t_{\hat{j}}^{\mathrm{fin}} \leq T \quad \frac{1}{k} \leq t_{\hat{j}}^{\mathrm{r}} \leq t_{\hat{j}}^{\mathrm{fin}}-t_{\hat{j}}^{\text {in }}+1
$$

By considering all feasible combinations of the three parameters $t^{\text {in }}$ (indexed with $x$ ), $t^{\text {fin }}$ (indexed with $y$ ) and $t^{\mathrm{r}}$, it follows:

$$
\hat{N} \leq \sum_{x=1}^{T} \sum_{y=x}^{T}(y-x+1) k=\frac{k}{6} T(T+1)(T+2) .
$$


Reminding that problem (27) has $T$ decision variables and $2 T+(T-1)+\hat{N}$ inequality constraints, we can choose the parameter $k$ in (21) to achieve the desired trade-off between computational complexity and clustering accuracy. If $k$ is sufficiently large, one can assume that the minimiser of (27), denoted by $\bar{\alpha}^{\diamond}$, reasonably approximates the solution $\bar{\alpha}^{*}$ of the original optimisation problem (17).

\section{Simulation Results}

The distributed coordination strategy and the clustering procedure are tested in simulation, considering a time interval of $24 \mathrm{~h}$ and a time discretisation step $\Delta t=0.48 \mathrm{~h}$. The UK power system is chosen for our case study, assuming that the inflexible demand $D_{i}$ corresponds to historical data of total power consumption in the grid, as measured by (National Grid, 2016). A significant penetration of flexible demand has been analysed, considering $N=$ $3 \cdot 10^{6}$ price-responsive appliances. All devices have equal rated power $P_{j}^{\mathrm{r}}=P^{\mathrm{r}}=6 \mathrm{KW}$ while their total required energy $E_{j}^{\mathrm{r}}$ is chosen according to a gaussian distribution with mean $\mu_{E}$ and standard deviation $\sigma_{E}$. This choice can correspond, for example, to a large fleet of electric vehicles that require to be fully charged and whose batteries have different state of charge when they are plugged into the grid. The initial availability times $t^{\text {in }}$ are generated according to a normal distribution with mean $\mu_{t}$ and standard deviation $\sigma_{t}$ (each value is rounded to the closest integer multiple of $\Delta t$ ). A similar procedure is adopted for $t_{j}^{\mathrm{fin}}=t_{j}^{\mathrm{in}}+\Delta_{j}$ where $\Delta_{j}$ follows a gaussian distribution with mean $\mu_{\Delta}$ and standard deviation $\sigma_{\Delta}$. The chosen values of the above parameters are:

$$
\begin{array}{ccc}
\mu_{E}=20 \mathrm{KWh} & \mu_{t}=23: 00 \mathrm{~h} & \mu_{\Delta}=13 \mathrm{~h} \\
\sigma_{E}=2 \mathrm{KWh} & \sigma_{t}=0.5 \mathrm{~h} & \sigma_{\Delta}=0.5 \mathrm{~h}
\end{array}
$$

\subsection{Performance Evaluation}

The clustering procedure is performed for $k=2$ : starting from $N=3 \cdot 10^{6}$ devices, a set $\hat{N}$ of macro players with cardinality $\hat{N}=566$ is derived. We choose $U(D)=0.5$. $m \cdot D^{2}+q D$ for the cost functional $J$ in (15), aiming at minimising the total generation costs of the system when the marginal cost of generation $\bar{C}(G)=m \cdot G+q$ is linear. As established in Remark 3 the signal $p(t)$ is equal to the price of inflexible demand, with $p(t)=\Pi\left(D_{i}(t)\right)$. The power constraint $\bar{\alpha}^{\diamond}$ is obtained by numerical resolution of (27), using the fmincon routine in a MATLAB environment. The optimal power constraint and the resulting demand profiles in the reordered time vector are shown in Fig. 3(a). Note that the aggregate demand $\bar{D}_{a}^{\diamond}$ is non decreasing, implying from Proposition 2 that a Nash equilibrium is obtained for the macro-appliances. The demand profiles and the proportional constraint as functions of time are shown in Fig. 3(b). With the proposed distributed scheduling, the appliances do not introduce a new demand peak and, on the contrary, induce a considerable valley-filling of the total power demand. The relationship between flexibility and energy costs is also analysed. Fig. 4 shows the average electricity price paid by a single appliance in the final system configuration as a function of its initial availability time $t^{\text {in }}$, for different values of $E^{\mathrm{r}}$. As mentioned in Remark 4, flexibility is incentivised and lower costs are achieved for lower values of $t^{\text {in }}$ (and larger availability).

The results presented so far have considered the reduced population of macroappliances obtained with the clustering described in Section 4. To quantify the approximation introduced by this procedure, we compare in Fig. 5 the flexible demand $\bar{D}_{f}^{\diamond}$ (total power consumption of the clustered loads) with the corresponding profile $\bar{D}_{f}$ obtained 
broadcasting $\bar{\alpha}^{\triangleright}$ to the original appliances population. Different values of the clustering parameter $k$ have been considered and only a subset of the considered time horizon, where differences are more consistent, is shown. For any $k \geq 1$ the discrepancy between demand profiles becomes negligible, suggesting that a Nash equilibrium should also be achieved by the original population. To analyse the impact of $k$, some relevant parameters are shown in Table $\mathrm{C} 1$. As specified before, $\hat{N}$ is the number of clustered appliances obtained from the original population of $N=3 \cdot 10^{6}$ devices. The quantity $T_{c}$ denotes the computational time required to solve the optimisation problem (27) with a HP Z600 machine equipped with an Intel Xeon CPU (frequency of 2.4Ghz) and 12GB of RAM. The mismatch between the aggregate power profile induced by the clustered and by the original population ( $D_{a}^{\diamond}$ and $D_{a}$, respectively) is quantified by the root mean square error $\delta_{c}=\sqrt{\sum_{t=1}^{T}\left(\bar{D}_{a}^{\diamond}(t)-\bar{D}_{a}(t)\right)^{2} / T}$.

To further evaluate the impact of the clustering procedure, this is applied to a small number $M=5000$ of macro-appliances approximating the original devices population. Given the reduced value of $M$, it is possible to numerically calculate the optimal power constraint $\bar{\alpha}_{\text {tr }}$ which minimises generation costs and induces an equilibrium for the macrodevices. The clustering is then applied, obtaining an approximate representation of the macro-appliances through $\hat{M}=244$ clustered loads. The optimal power constraint $\bar{\alpha}_{\mathrm{ag}}$ for this reduced population is also calculated. To quantify the performance degradation introduced by the clustering, the results obtained broadcasting $\bar{\alpha}_{\text {tr }}$ and $\bar{\alpha}_{\text {ag }}$ to the initial population of $M=5000$ macro-appliances are compared. It can be verified that total generation costs and the average costs sustained by the single load differ by $0.0195 \%$ and $0.0312 \%$, respectively. We wish to emphasise that such negligible discrepancies are expected to be even smaller for higher values of $M$ (for example in realistic cases of large-scale flexible demand deployment) as the granularity of the devices parameters within the population is reduced and the clustered representation becomes more accurate.

\subsection{Comparison with Alternative Control Schemes}

The proposed control technique (hereby denoted as 'OS' - Optimal Scheduling) is also compared to other strategies that do not consider a proportional power constraint or global optimisation. In the scenario 'UCM' (Unconstrained Cost Minimisation) each device determines its cost-minimising scheduling on the basis of the broadcast price signal $p=\Pi\left(D_{i}\right)$, with no limitations on the power rate ( $\alpha$ identically equal to 1$)$. In the second alternative scheme, denoted by 'GPA' (Greedy Power Allocation), all devices aim at completing their tasks as soon as possible and operate (at maximum power) from the initial availability instant $t_{j}^{\text {in }}$ until task completion. The aggregate demand profiles in the three cases (respectively $D_{a}^{\mathrm{OS}}, D_{a}^{\mathrm{UCM}}$ and $D_{a}^{\mathrm{GPA}}$ ) are shown in Fig. 6. From a global point of view, the UCM and GPA strategies do not guarantee any valley filling. On the contrary, they introduce a new demand peak of considerable entity. Such peak occurs at time instants with lowest inflexible demand (in the UCM case) or right after most of the devices have become available (in the GPA case). It is straightforward to verify that a Nash equilibrium is not achieved with the UCM and GPA schemes since most of the devices could reduce their energy cost by shifting part of their power consumption to the valleys that arise in the profiles of aggregate demand. Some quantities of interest for the techniques mentioned above are compared in Table C2: the global cost function $J$ (i.e. total generation costs), the average energy $\operatorname{cost} C_{\mathrm{av}}$ sustained by the single device and the average final time instant $t_{\mathrm{av}}^{\mathrm{fin}}$ of power consumption. As expected, the proposed OS strategy guarantees the lowest value of $J$ and it also minimises $C_{\text {av }}$. The higher values of average energy cost $C_{\mathrm{av}}$ in the UCM and GPA scenarios are a direct result of the power scheduling of the de- 
vices. In these cases, all loads follow similar strategies, consistently increasing the final demand values at the times during which they operate, thus buying electricity at higher prices. There is also a clear trade-off between the costs sustained by the devices and their final times of operation: the introduction of a proportional power constraint in the OS case allows to reduce the energy cost but it also implies longer task times, since occasionally the devices will not operate at their maximum rated power.

\section{Properties of the Optimal Power Constraint}

Closed-form calculation of the optimal solution $\bar{\alpha}^{*}$ for the original scheduling problem (17) is in general extremely difficult. In this section a candidate solution $\bar{\alpha}^{\star}$ is analytically characterised, discussing its correspondence with the numerical solution and its optimality properties. Specifically, it is shown that each feasible constraint signal $\bar{\alpha}$ can be associated, under certain assumptions, to a vector of constrained task times $t^{\text {end }}$ (time required by each appliance to complete its task under the chosen power constraint) and to the state trajectory of a dynamical system integrated backward in time. Using this analysis as a starting point, structural properties of the candidate solution $\bar{\alpha}^{\star}$ are derived in Proposition 4 , underlying its correspondence with the simulative results. The main result of this section, presented in Theorem 1, shows that $\bar{\alpha}^{\star}$ achieves Pareto optimality for the task times of the appliances.

\subsection{Power Constraint as Solution of a Dynamical System}

Some quantities are preliminarily introduced to characterise the scheduled power of the individual appliance and define it with a simplified expression.

Definition 3 Consider $\bar{\alpha}: \mathscr{T} \rightarrow[0,1]$ which is feasible for (17) and the resulting $\bar{u}_{j}^{*}(\cdot, \bar{\alpha})$ introduced in (6). We define as $t_{j}^{\text {end }}$ the maximum $t \in \mathscr{T}$ such that $\bar{u}_{j}^{*}(t, \bar{\alpha})=\bar{\alpha}(t) \cdot P_{j}^{\mathrm{r}}$, corresponding to the first case in expression (7). Similarly, $t_{j}^{\text {res }}$ is the unique $t$ for which $\bar{u}_{j}^{*}(t, \bar{\alpha})=\left(t_{j}^{\mathrm{r}}-\bar{\alpha}_{j}^{I}(t-1)\right) \Delta t^{-1} P_{j}^{\mathrm{r}}$. This last quantity is denoted by $P_{j}^{\text {res }}$.

The quantities $t_{j}^{\text {end }}$ and $t_{j}^{\text {res }}$ are always well defined given the monotonicity of $\bar{\alpha}_{j}^{I}$, appearing in (7) and defined in (8). The $j$-th device operates at maximum feasible power at the time instants $\left\{0,1, \ldots, t_{j}^{\text {end }}\right\} \cap \overline{\mathscr{A}}_{j}$ and consumes the last required power $P_{j}^{\text {res }}$ (lower than the maximum feasible value) at $t_{j}^{\text {res }}$, with $t_{j}^{\text {res }}>t_{j}^{\text {end }}$. The quantity $t_{j}^{\text {end }}$ is now considered as the final time of power consumption in the reordered time vector, i.e. the constrained task time of the $j$-th appliance (time required to complete the task under the specified power constraint). This allows to provide a simplified expression for $\bar{u}^{*}(\cdot, \bar{\alpha})$ :

$$
\bar{u}_{j}^{*}(t, \bar{\alpha})=\left\{\begin{array}{cc}
\bar{\alpha}(t) \cdot P_{j}^{\mathrm{r}} \text { if } t \in\left\{1, \ldots, t_{j}^{\text {end }}\right\} \\
0 & \text { otherwise }
\end{array}\right.
$$

This formulation requires adjustments on the inflexible demand profile (incorporating the power consumption of all devices at their last time instant of operation) and on the rated 
task time of the appliances (discounting the residual power consumption $P_{J}^{\text {res }}$ ):

$$
\begin{array}{rlrl}
\hat{D}_{i}(t) & =\bar{D}_{i}(t)+\sum_{\left\{j: t_{j}^{\mathrm{res}}=t\right\}} P_{j}^{\mathrm{res}} & & \forall t \in \mathscr{T} \\
\hat{t}_{j}^{\mathrm{r}} & =t_{j}^{\mathrm{r}}-\frac{P_{j}^{\mathrm{res}} \Delta t}{P_{j}^{\mathrm{r}}} & \forall j \in \mathscr{N} .
\end{array}
$$

Assumption 4 The appliances parameters are such that the following approximation can be considered:

$$
\hat{D}_{i} \simeq \bar{D}_{i} \quad \hat{t}_{j}^{r} \simeq t_{j}^{r} \quad \forall j \in \mathscr{N} .
$$

This is reasonable to assume for small values of $\Delta t$ (the energy $P_{j}^{\text {res }} \cdot \Delta t$ consumed at $t_{j}^{\text {res }}$ becomes negligible) and if the final times $t_{j}^{\text {end }}$ are sufficiently diversified (power variations in $\hat{D}_{i}$ will not occur at the same time instants). As a result, the adjusted inflexible profile $\hat{D}_{i}$ and task times $\hat{t}_{j}^{\mathrm{r}}$, which depend in general on the specific choice of the constraint $\bar{\alpha}$, can always be approximated by their original values (respectively $\bar{D}_{i}$ and $t_{j}^{\mathrm{r}}$ ). Therefore, it is possible to operate in the current modelling framework using the simplified expression (29), rather than (7), to characterise $\bar{u}^{*}$. The validity of Assumption 4 has been tested for the case study presented in Section 5, deriving the following results:

$$
\frac{1}{T} \sum_{t=1}^{T} \frac{\left|\hat{D}_{i}(t)-\bar{D}_{i}(t)\right|}{\bar{D}_{i}(t)}=0.0018 \quad \frac{1}{N} \sum_{j=1}^{N} \frac{\left|\hat{t}_{j}^{\mathrm{r}}-t_{j}^{\mathrm{r}}\right|}{t_{j}^{\mathrm{r}}}=0.0139 .
$$

Note that the mean normalised error on the rated task times of the appliances, with the proposed approximation, is equal to about $1 \%$. The error is one order of magnitude smaller when the inflexible demand is considered, leading to conclude that the approximations in (31) are valid for the simulations of Section 5. Considering also that such discrepancies can be further reduced by choosing lower time steps, it is reasonable to conclude that Assumption 4 and the results presented in this section can in general be applied to realistic contexts.

Definition 4 Given a function $\bar{g}: \mathscr{T} \rightarrow \mathrm{R}$ in the reordered time vector, as described in Definition 1, let $\tilde{g}: \mathscr{T} \rightarrow \mathrm{R}$ denote (with tilde superscript) the corresponding function backward in time:

$$
\tilde{g}(t)=\bar{g}(T-t+1) \quad \forall t \in \mathscr{T} .
$$

The correspondence also holds in the opposite sense, with $\bar{g}(t)=\tilde{g}(T-t+1)$.

For a fixed parameter vector $t^{\text {end }}=\left[t_{1}^{\text {end }}, \ldots, t_{N}^{\text {end }}\right]$, we indicate by $\tilde{\mathscr{A}}_{j}$ the set of time instants (in the backward coordinates) during which the $j$-th device is consuming power:

$$
\tilde{\mathscr{A}}_{j}=\left\{t:(T-t+1) \in\left(\overline{\mathscr{A}}_{j} \cap\left\{1, \ldots, t_{j}^{\text {end }}\right\}\right)\right\} .
$$

Let $P^{\text {av }}\left(t, t^{\text {end }}\right)$ denote the maximum power that, for the current final times $t^{\text {end }}$ and resulting sets $\tilde{\mathscr{A}}_{j}$, can be consumed by the appliances available at time $t$ :

$$
P^{\mathrm{av}}\left(t, t^{\mathrm{end}}\right)=\sum_{\left\{j: t \in \tilde{A}_{j}\right\}} P_{j}^{\mathrm{r}}
$$


Adopting the notation and concepts introduced above, any candidate solution of (17) can be characterised through a discrete-time dynamical system with state $\left[\tilde{\phi}_{D}, \tilde{\phi}_{\alpha_{1}}, \ldots, \tilde{\phi}_{\alpha_{N}}\right]$ :

$$
\begin{array}{rlrl}
\tilde{\phi}_{D}(t) & =\tilde{\phi}_{D}(t-1)+\tilde{F}(t) & & \tilde{\phi}_{D}(1)=0 \\
\tilde{\phi}_{\alpha_{j}}(t) & =\tilde{\phi}_{\alpha_{j}}(t-1)+\tilde{\alpha}(t) \mathbb{1}_{\tilde{\mathscr{A}}_{j}}(t) & \tilde{\phi}_{\alpha_{j}}(1)=0 & j=1, \ldots, N
\end{array}
$$

The following relationship is also imposed between $\tilde{F}$ and the proportional constraint $\tilde{\alpha}$ :

$$
\tilde{\phi}_{D}(t-1)+\tilde{F}(t)=\tilde{\alpha}(t) P^{\mathrm{av}}\left(t, t^{\mathrm{end}}\right) .
$$

The interpretation of (35) is twofold and depends on which variable is considered as control input (respectively $\tilde{\alpha}$ or $\tilde{F}$ ). In the former case, given a feasible $\bar{\alpha}$ for (17) and the corresponding quantities presented in Definition 3 and 4, by considering (36) and (29) we have:

$$
\begin{aligned}
\tilde{\phi}_{D}(t) & =\tilde{\phi}_{D}(t-1)+\tilde{F}(t)=\tilde{\alpha}(t) \sum_{\left\{j: t \in \tilde{\mathscr{A}}_{j}\right\}} P_{j}^{\mathrm{r}} \\
& =\bar{\alpha}(T-t+1) \sum_{\left\{j: T-t+1 \in \overline{\mathscr{A}}_{j} \cap\left\{1, \ldots, t_{j}^{\text {end }}\right\}\right\}} P_{j}^{\mathrm{r}} \\
& =\sum_{j=1}^{N} \bar{u}_{j}^{*}(T-t+1, \bar{\alpha})=\bar{D}_{f}(T-t+1)=\tilde{D}_{f}(t) .
\end{aligned}
$$

This means that the dynamical system (35) keeps track, in one of its state components, of flexible demand $\tilde{D}_{f}$ (backward in time). Similarly, it is possible to show that $\tilde{\phi}_{\alpha_{j}}(t) \cdot P_{j}^{\mathrm{r}}$ corresponds to the amount of power consumed by the $j$-th appliance in the (forward) time interval $\{T-t+1, T-t+2 \ldots, T-1, T\}$. Therefore, for a feasible $\bar{\alpha}$, it must hold:

$$
\tilde{\phi}_{\alpha_{j}}(T)=\frac{E_{j}^{\mathrm{r}}}{P_{j}^{\mathrm{r}}}=t_{j}^{\mathrm{r}} \quad j=1, \ldots, N
$$

If the parameters $t^{\text {end }}=\left[t_{1}^{\text {end }}, \ldots, t_{N}^{\text {end }}\right]$ are fixed a priori, it is also possible to consider $\tilde{F}$ as the control input of system (35) and derive the corresponding $\tilde{\alpha}$ from (36):

$$
\tilde{\alpha}(t)=\frac{\tilde{\phi}_{D}(t-1)+\tilde{F}(t)}{\sum_{\left\{j: t \in \tilde{A}_{j}\right\}} P_{j}^{\mathrm{r}}}=\frac{\tilde{\phi}_{D}(t-1)+\tilde{F}(t)}{P^{\mathrm{av}}\left(t, t^{\text {end }}\right)} .
$$

The following feasibility result can now be provided:

Proposition 3 For a fixed parameter vector $t^{\text {end }}$ and input signal $\tilde{F}: \mathscr{T} \rightarrow \mathrm{R}$ for system (35), consider the resulting $\tilde{\alpha}$ calculated according to (39) and the equivalent quantity $\bar{\alpha}$ forward in time, with $\bar{\alpha}(t)=\tilde{\alpha}(T-t+1)$ as specified in Definition 4. If $\bar{\alpha}$ is feasible for the optimisation problem (17), the following inequalities must be satisfied:

$$
\begin{array}{ll}
\tilde{F}(t) \leq P^{\mathrm{av}}\left(t, t^{\mathrm{end}}\right)-\tilde{\phi}_{D}(t-1) & \forall t \in\{1, \ldots, T\} \\
\tilde{F}(t) \leq \tilde{D}_{i}(t-1)-\tilde{D}_{i}(t) & \forall t \in\{2, \ldots, T\} .
\end{array}
$$

Proof. From (39), the right inequality in (17b) can be rewritten as:

$$
\bar{\alpha}(t)=\tilde{\alpha}(T-t+1)=\frac{\tilde{\phi}_{D}(T-t)+\tilde{F}(T-t+1)}{P^{\mathrm{av}}\left(T-t+1, t^{\text {end }}\right)} \leq 1 \quad \forall t \in\{1, \ldots, T\} .
$$


Multiplying by $P^{\mathrm{av}}$ and subtracting $\tilde{\phi}_{D}(T-t)$ from both sides yields equivalence to (40a). Similarly the constraint (17c), considering Definition 4 and (37), is equivalent to:

$$
\tilde{D}_{i}(t)+\tilde{\phi}_{D}(t) \leq \tilde{D}_{i}(t-1)+\tilde{\phi}_{D}(t-1) \quad \forall t \in\{2, \ldots, T\}
$$

By replacing $\tilde{\phi}_{D}(t)$ with its expression in (35) and rearranging the terms of inflexible demand it is straightforward to obtain (40b).

\subsection{Candidate Power Constraint: Definition and Properties}

The following candidate solution is now considered for the optimisation problem (17):

Definition 5 Fixed the parameter vector $t^{\text {end }}=t^{\star}=\left[t_{1}^{\star}, \ldots, t_{N}^{\star}\right]$, define the following feedback law:

$$
\tilde{\psi}\left(t, \tilde{\phi}_{D}\right)=\min \left\{P^{\mathrm{av}}\left(t, t^{\star}\right)-\tilde{\phi}_{D}, \tilde{D}_{i}(t-1)-\tilde{D}_{i}(t)\right\}
$$

Let $\tilde{\phi}^{\star}=\left[\tilde{\phi}_{D}^{\star}, \tilde{\phi}_{\alpha_{1}}^{\star}, \ldots, \tilde{\phi}_{\alpha_{N}}^{\star}\right]$ denote the solution of system (35) when $\tilde{F}(t)=\tilde{F}^{\star}(t)=$ $\tilde{\psi}\left(t, \tilde{\phi}_{D}(t-1)\right)$ and $\tilde{\alpha}^{\star}$ the corresponding result of (39). The equivalent function forward in time is denoted by $\bar{\alpha}^{\star}$, as specified in Definition 4 with $\bar{g}=\bar{\alpha}^{\star}$.

To better characterise the quantities introduced above and to show their relationship with the simulations presented in the previous section, the following result is provided:

Proposition 4 Denote by $\tilde{\phi}^{\circ}=\left[\tilde{\phi}_{D}^{\circ}, \tilde{\phi}_{\alpha_{1}}^{\circ}, \ldots, \tilde{\phi}_{\alpha_{N}}^{\circ}\right]$ the solution of (35) for some input $\tilde{F}=\tilde{F}^{\circ}$ fulfilling (40). Let $\tilde{\alpha}^{\circ}$ be the resulting proportional constraint defined by (39) and $\tilde{D}_{a}$ the corresponding aggregate demand. The following conditions are verified:

$$
\begin{array}{ll}
\tilde{D}_{a}(t) \leq \tilde{D}_{a}(t-1) & \forall t: \tilde{\alpha}^{\circ}(t)=1 \\
\tilde{D}_{a}(t)=\tilde{D}_{a}(t-1) & \forall t: \tilde{\alpha}^{\circ}(t)<1
\end{array}
$$

if and only if $\tilde{F}^{\circ}=\tilde{F}^{\star}$ as specified in Definition 5 .

Proof. See B.

The sufficient part of the proposition statement shows that (42) holds when $\tilde{F}^{\star}$ and $\tilde{\alpha}^{\star}$ in Definition 5 are applied. The necessary part, on the other hand, implies that when (42) holds for the simulative results, the solution obtained by numerically solving the global optimisation problem (17) corresponds to the one introduced in Definition 5. This is true, for example, in the case studies presented in Section 5. One can consider the set of conditions forward in time equivalent to (42):

$$
\begin{array}{ll}
\bar{D}_{a}(t) \leq \bar{D}_{a}(t+1) & \forall t: \bar{\alpha}^{\circ}(t)=1 \\
\bar{D}_{a}(t)=\bar{D}_{a}(t+1) & \forall t: \bar{\alpha}^{\circ}(t)<1
\end{array}
$$

and verify that these are fulfilled in Fig. 3(a). For an easier check, the values of aggregate power demand and proportional power constraint obtained in simulation are plotted, over a significant time interval, in Fig. 7. Note that the mentioned correspondence has been verified to hold for all the other numerical simulations that have been performed. 


\subsection{Pareto Optimality}

It is now proved that the power scheduling induced by $\bar{\alpha}=\bar{\alpha}^{\star}$ is Pareto optimal with respect to the task times of the appliances.

Theorem 1 Assume that, for the chosen final time parameters $t^{\star}$, the proportional constraint $\bar{\alpha}^{\star}$ introduced in Definition 5 is feasible for problem (17). Let $\bar{\alpha}^{\circ}:[0,1] \rightarrow \mathscr{T}$ be a distinct candidate solution. If, for the corresponding final time parameters $t^{\text {end }}=t^{\circ}$ introduced in Definition 3, it holds:

$$
\begin{gathered}
t_{j}^{\circ} \leq t_{j}^{\star} \quad \forall j \in \mathscr{N} \\
\exists \hat{j} \in \mathscr{N}: t_{\hat{j}}^{\circ}<t_{\hat{j}}^{\star}
\end{gathered}
$$

then the function $\bar{\alpha}^{\circ}$ is infeasible for (17).

Proof. See C.

We can conclude that, when $\bar{\alpha}^{\star}$ is feasible, there is no other $\bar{\alpha}^{\circ}$ which induces a Nash equilibrium and reduces the task time of some appliances without degrading the performance of other devices. From previous considerations, this result should in principle hold also for the numerical solution of (17), such as the one presented in the simulation section.

\section{Conclusions}

This paper presents a novel distributed control scheme for the coordination of priceresponsive appliances with partial flexibility. A price signal and a proportional constraint are broadcast to the devices, which independently schedule their power consumption in order to minimise their energy cost. The broadcast quantities achieve one-step convergence to a Nash equilibrium (characterised through monotonicity of demand in reordered coordinates) and optimise some global index, such as total generation costs. A clustering of the devices is introduced to reduce the computational complexity of the optimisation which is then solved numerically. Finally, the performance of the stable optimal solution is evaluated in simulations and a theoretical analysis is carried out to determine its optimality properties with respect to the task times of the appliances.

\section{References}

Albadi, M., \& El-Saadany, E. (2008). A summary of demand response in electricity markets. Electric Power Systems Research, 78(11), 1989-1996. doi:10.1016/j.epsr.2008.04.002.

Boait, P., Ardestani, B. M., \& Snape, J. R. (2013). Accommodating renewable generation through an aggregator-focused method for inducing demand side response from electricity consumers. IET Renewable Power Generation, 7(6), 689-699. doi:10.1049/iet-rpg.2012.0229.

Chen, H., Li, Y., Louie, R., \& Vucetic, B. (2014). Autonomous demand side management based on energy consumption scheduling and instantaneous load billing: An aggregative game approach. IEEE Transactions on Smart Grid, 5(4), 1744-1754. doi:10.1109/TSG.2014.2311122.

Chen, Z., Wu, L., \& Fu, Y. (2012). Real-time price-based demand response management for residential appliances via stochastic optimization and robust optimization. IEEE Transactions on Smart Grid, 3(4), 1822-1831. doi:10.1109/TSG.2012.2212729. 
De Paola, A., Angeli, D., \& Strbac, G. (2015). Analysis of Nash equilibria in energy markets with large populations of price-responsive flexible appliances. 2015 54th IEEE Conference on Decision and Control (CDC), 5587-5592. doi:10.1109/CDC.2015.7403095.

De Paola, A., Angeli, D., \& Strbac, G. (2016). Integration of price-responsive appliances in the energy market through flexible demand saturation. IEEE Transactions on Control of Network Systems, PP(99), doi:10.1109/TCNS.2016.2583204.

Fan, Z. (2012). A distributed demand response algorithm and its application to PHEV charging in smart grids. IEEE Transactions on Smart Grid, 3(3), 1280-1290. doi:10.1109/TSG.2012.2185075.

Gan, L., Topcu, U., \& Low, S. H. (2013). Optimal decentralized protocol for electric vehicle charging. IEEE Transactions on Power Systems, 28(2), 940-951. doi:10.1109/TPWRS.2012.2210288.

Grammatico, S., Parise, F., Colombino, M., \& Lygeros, J. (2016). Decentralized convergence to Nash equilibria in constrained deterministic mean field control. IEEE Transactions on Automatic Control, 61(11), 3315-3329. doi:10.1109/TAC.2015.2513368.

Lasry, J., \& Lions, P. (2007). Mean field games. Japanese Journal of Mathematics, 2(1), 229-260. doi:10.1007/s11537-007-0657-8.

Ma, Z., Callaway, D., \& Hiskens, I. (2013). Decentralized charging control of large populations of plug-in electric vehicles. IEEE Transactions on Control Systems Technology, 21(1), 67-78. doi:10.1109/TCST.2011.2174059.

Ma, Z., Zou, S., Ran, L., Shi, X., \& Hiskens, I. (2016). Efficient decentralized coordination of large-scale plug-in electric vehicle charging. Automatica, 69, 35-47. doi:10.1016/j.automatica.2016.01.035.

Mohsenian-Rad, H., Wong, V. W. S., Jatskevich, J., Schober, R., \& Leon-Garcia, A. (2010). Autonomous demand-side management based on game-theoretic energy consumption scheduling for the future smart grid. IEEE Transactions on Smart Grid, 1(3), 320-331. doi:10.1109/TSG.2010.2089069.

National Grid. (2016, June). Historical demand data. Retrieved from http: / / www2.nationalgrid.com/UK/Industry-information/ Electricity-transmission-operational-data/Data-Explorer/

Olivier, G., Lasry, J. M., \& Lions, P. L. (2011). Mean field games and applications. In Paris-Princeton lectures on mathematical finance 2010 (pp. 205-266). Berlin, Heidelberg: Springer Berlin Heidelberg.

Papadaskalopoulos, D., \& Strbac, G. (2013). Decentralized participation of flexible demand in electricity markets - part I: Market mechanism. IEEE Transactions on Power Systems, 28(4), 3658-3666. doi:10.1109/TPWRS.2013.2245686.

Ruthe, S., Rehtanz, C., \& Lehnhoff, S. (2015). On the problem of controlling shiftable prosumer devices with price signals. International Journal of Electrical Power \& Energy Systems, 72, 83-90. doi:10.1016/j.ijepes.2015.02.014.

Samadi, P., Mohsenian-Rad, H., Schober, R., \& Wong, V. W. S. (2012). Advanced demand side management for the future smart grid using mechanism design. IEEE Transactions on Smart Grid, 3(3), 1170-1180. doi:10.1109/TSG.2012.2203341.

Strbac, G. (2008). Demand side management: Benefits and challenges. Energy Policy, 36(12), 4419-4426. doi:10.1016/j.enpol.2008.09.030.

Su, C. L., \& Kirschen, D. (2009). Quantifying the effect of demand response on electricity markets. IEEE Trans. on Power System, 24(3), 1199-1207. doi:10.1109/TPWRS.2009.2023259.

\section{Appendix A. Proof of Proposition 1}

For the feasibility of $\bar{u}_{j}^{*}$, consider that (3) in Lemma 1 is equivalent to $\bar{\alpha}_{j}^{I}(T) \geq E_{j}^{\mathrm{r}} / P_{j}^{\mathrm{r}}=t_{j}^{\mathrm{r}}$. Given the monotonicity of $\bar{\alpha}_{j}^{I}$, guaranteed by $\bar{\alpha}(l)$ always taking values in the interval $[0,1]$, there is a unique $x \in \overline{\mathscr{A}}_{j}$ such that $\bar{\alpha}_{j}^{I}(x-1)<t_{j}^{\mathrm{r}} \leq \bar{\alpha}_{j}^{I}(x)$ and for which the second case in (7) must be considered when evaluating $\bar{u}_{j}^{*}(x, \bar{\alpha})$. Subtracting $\bar{\alpha}_{j}^{I}(x-1)$ from all 
terms of the inequality above and dividing by $\Delta t$ yields $0 \leq\left(t_{j}^{\mathrm{r}}-\bar{\alpha}_{j}^{I}(x-1)\right) / \Delta t \leq \bar{\alpha}(x)$. Therefore, all three cases in expression (7) always satisfy the inequality constraints in (6) for $\bar{u}=\bar{u}_{j}^{*}$. It is straightforward to verify that also the equality constraint is fulfilled:

$$
\begin{aligned}
\sum_{t=1}^{T} \bar{u}_{j}^{*}(t, \bar{\alpha}) \Delta t & =P_{j}^{\mathrm{r}}\left[\sum_{t \in\left(\bar{\alpha}_{j} \cap\{1, \ldots, x-1\}\right)} \bar{\alpha}(t) \Delta t+\left(t_{j}^{\mathrm{r}}-\bar{\alpha}_{j}^{I}(x-1)\right)\right] \\
& =P_{j}^{\mathrm{r}} \cdot\left[\bar{\alpha}_{j}^{I}(x-1)+\left(t_{j}^{\mathrm{r}}-\bar{\alpha}_{j}^{I}(x-1)\right)\right]=E_{j}^{\mathrm{r}} .
\end{aligned}
$$

To prove the optimality of $\bar{u}^{*}$, note that all power profiles that are feasible for (6) have fixed total sum. Therefore, any $\bar{u}$ fulfilling the constraints in (6) can be written as $\bar{u}=$ $\bar{u}^{*}+\sum_{l=1}^{L} \delta_{l}$, i.e. as the sum of $\bar{u}^{*}$ and $L$ finite variations $\delta_{l}$, with the following structure:

$$
\delta_{l}(t)=\left\{\begin{array}{ccc}
-\varepsilon & \text { if } & t=x_{1} \\
\varepsilon & \text { if } & t=x_{2} \\
0 & & \text { otherwise }
\end{array}\right.
$$

Moreover, assuming $\varepsilon>0$, the following conditions can be imposed on $x_{1}$ and $x_{2}$ :

$$
1 \leq x_{1} \leq x \leq x_{2} \leq T
$$

This is true since $\bar{u}^{*}$ corresponds to the maximum feasible value of $\bar{u}$ for $t<x$. Given the monotonicity of the price $\bar{p}$ in the reordered time vector, it holds:

$$
\sum_{t=1}^{T} \bar{p}(t) \delta_{l}(t) \Delta t=\varepsilon\left[\bar{p}\left(x_{2}\right)-\bar{p}\left(x_{1}\right)\right] \Delta t>0 \quad \forall l \in\{1, \ldots, L\} .
$$

Optimality of $\bar{u}^{*}$ for (6) follows from the linearity of the cost function with respect to $\bar{u}=\bar{u}^{*}+\sum_{l=1}^{L} \delta_{l}$.

\section{Appendix B. Proof of Proposition 4}

Condition (42a) is analysed first. As a result of expression (39), the equality $\tilde{\alpha}^{\circ}(t)=1$ can be rewritten as:

$$
\tilde{\phi}_{D}^{\circ}(t-1)+\tilde{F}^{\circ}(t)=P^{\mathrm{av}}\left(t, t^{\mathrm{end}}\right) .
$$

Given the state equations (35) and conditions (36)-(37), it holds:

$$
\tilde{D}_{f}(t)=\tilde{\phi}_{D}^{\circ}(t)=\tilde{\phi}_{D}^{\circ}(t-1)+\tilde{F}^{\circ}(t)=P^{\mathrm{av}}\left(t, t^{\mathrm{end}}\right) .
$$

This means that $\tilde{F}^{\circ}$ has an unequivocal expression:

$$
\tilde{F}^{\circ}(t)=P^{\mathrm{av}}\left(t, t^{\mathrm{end}}\right)-\tilde{\phi}_{D}^{\circ}(t-1) \leq \tilde{D}_{i}(t-1)-\tilde{D}_{i}(t)
$$

where the inequality follows from condition (40b). To see that (42a) is fulfilled, it is sufficient to show the following:

$$
\tilde{D}_{a}(t)=\tilde{D}_{i}(t)+\tilde{D}_{f}(t-1)+\tilde{F}^{\circ}(t) \leq \tilde{D}_{i}(t-1)+\tilde{D}_{f}(t-1)=\tilde{D}_{a}(t-1) .
$$


With similar considerations it is possible to check that, when $\tilde{\alpha}^{\circ}<1$, we have:

$$
\tilde{F}^{\circ}(t)<P^{\mathrm{av}}\left(t, t^{\mathrm{end}}\right)-\tilde{\phi}_{D}^{\circ}(t-1) .
$$

Given (37) and (39), condition (42b) is verified if and only if:

$$
\begin{aligned}
\tilde{F}^{\circ}(t) & =\tilde{\phi}_{D}^{\circ}(t)-\tilde{\phi}_{D}^{\circ}(t-1)=\tilde{D}_{f}(t)-\tilde{D}_{f}(t-1) \\
& =\tilde{D}_{a}(t)-\tilde{D}_{i}(t)-\tilde{D}_{a}(t-1)+\tilde{D}_{i}(t-1)=\tilde{D}_{i}(t-1)-\tilde{D}_{i}(t) .
\end{aligned}
$$

By combining the previous results, we have:

$$
\begin{array}{cc}
\tilde{F}^{\circ}(t)=P^{\mathrm{av}}\left(t, t^{\mathrm{end}}\right)-\tilde{\phi}_{D}^{\circ}(t-1) & \forall t: \tilde{\alpha}^{\circ}(t)=1 \\
P^{\mathrm{av}}\left(t, t^{\text {end }}\right)-\tilde{\phi}_{D}^{\circ}(t-1) \leq \tilde{D}_{i}(t-1)-\tilde{D}_{i}(t) & \\
\tilde{F}^{\circ}(t)=\tilde{D}_{i}(t-1)-\tilde{D}_{i}(t) & \forall t: \tilde{\alpha}^{\circ}(t)<1 \\
\tilde{D}_{i}(t-1)-\tilde{D}_{i}(t) \leq P^{\mathrm{av}}\left(t, t^{\text {end }}\right)-\tilde{\phi}_{D}^{\circ}(t-1) &
\end{array}
$$

The proof is concluded by noticing that (B4) is equivalent to $\tilde{F}^{\circ}(t)=\tilde{\psi}\left(t, \tilde{\phi}_{D}(t-1)\right)=$ $\tilde{F}^{\star}(t)$ as defined in $(41)$.

\section{Appendix C. Proof of Theorem 1}

We denote by $\tilde{\phi}^{\circ}=\left[\tilde{\phi}_{D}^{\circ}, \tilde{\phi}_{\alpha_{1}}^{\circ}, \ldots, \tilde{\phi}_{\alpha_{N}}^{\circ}\right]$ the solution of the dynamical system (35) when $\tilde{\alpha}=\tilde{\alpha}^{\circ}$. Similarly, $\tilde{F}^{\circ}$ corresponds to the input signal $\tilde{F}$ which fulfils (36) for $\tilde{\alpha}=\tilde{\alpha}^{\circ}$, $\tilde{\phi}_{D}=\tilde{\phi}_{D}^{\circ}$ and $t^{\text {end }}=t^{\circ}$. If the power constraint $\bar{\alpha}^{\circ}$ is feasible, the following must hold:

$$
\begin{aligned}
& \sum_{t=1}^{T} \tilde{\phi}_{D}^{\circ}(t)=\sum_{t=1}^{T} \tilde{\phi}_{D}^{\star}(t) \\
& \tilde{\phi}_{\alpha_{j}}^{\circ}(T)=\tilde{\phi}_{\alpha_{j}}^{\star}(T)=t_{j}^{\mathrm{r}} \quad \forall j \in \mathscr{N}
\end{aligned}
$$

where $\tilde{\phi}^{\star}=\left[\tilde{\phi}_{D}^{\star}, \tilde{\phi}_{\alpha_{1}}^{\star}, \ldots, \tilde{\phi}_{\alpha_{N}}^{\star}\right]$ represents the solution of system (35) when $\tilde{F}^{\star}$ is applied, as specified in Definition 5. From (37), condition (C1a) must hold since the total flexible demand in the system is fixed and corresponds to the total sum of $\tilde{\phi}_{D}^{\star}$ (given that $\bar{\alpha}^{\star}$ is assumed feasible in the theorem statement). Similarly, the equalities in (C1b) follow from the feasibility of $\bar{\alpha}^{\star}$ and (38). We initially show that $\tilde{\phi}_{D}^{\circ}(t)-\tilde{\phi}_{D}^{\star}(t) \leq 0$ for all $t \in \mathscr{T}$. Since $\tilde{\phi}_{D}^{\circ}(1)=\tilde{\phi}_{D}^{\star}(1)=0$, it is sufficient to prove the following when $t>0$ and $\tilde{\phi}_{D}^{\circ}(t-$ $1)-\tilde{\phi}_{D}^{\star}(t-1) \leq 0$ :

$$
\tilde{\phi}_{D}^{\circ}(t)-\tilde{\phi}_{D}^{\star}(t)=\tilde{\phi}_{D}^{\circ}(t-1)+\tilde{F}^{\circ}(t)-\tilde{\phi}_{D}^{\star}(t-1)-\tilde{F}^{\star}(t) \leq 0 .
$$

Given (44) and expression (34) for $P^{\text {av }}$, it follows:

$$
\begin{gathered}
P^{\mathrm{av}}\left(t, t^{\circ}\right) \leq P^{\mathrm{av}}\left(t, t^{\star}\right) \quad \forall t \in \mathscr{T} \\
P^{\mathrm{av}}\left(x, t^{\circ}\right)<P^{\mathrm{av}}\left(x, t^{\star}\right)
\end{gathered}
$$

where $x=T-t_{\hat{j}}^{\star}+1$ represents the final time parameter of the $\hat{j}$-th device in the backward coordinates, with $t_{\hat{j}}^{\circ}<t_{\hat{j}}^{\star}$ as specified in the theorem statement. From (C3), considering 
Proposition 3 for $\tilde{F}^{\circ}(t)$ and expression (41) for $\tilde{F}^{\star}(t)=\tilde{\psi}\left(t, \tilde{\phi}_{D}^{\star}(t-1)\right)$, the following holds when $\tilde{\phi}_{D}^{\circ}(t-1)-\tilde{\phi}_{D}^{\star}(t-1) \leq 0$ :

$$
\tilde{F}^{\circ}(t)-\tilde{F}^{\star}(t) \leq \tilde{\phi}_{D}^{\star}(t-1)-\tilde{\phi}_{D}^{\circ}(t-1) .
$$

This means that $(\mathrm{C} 2)$ is always fulfilled and therefore $\tilde{\phi}_{D}^{\circ}(t)-\tilde{\phi}_{D}^{\star}(t) \leq 0$ for all $t \in \mathscr{T}$. Two cases must now be considered: if there exists $\tilde{t} \in \mathscr{T}$ such that $\tilde{\phi}_{D}^{\circ}(\tilde{t})<\tilde{\phi}_{D}^{\star}(\tilde{t})$ then condition (C1a) is violated. Alternatively, $\phi_{D}^{\circ}$ and $\phi_{D}^{\star}$ are identically equal. To show that also in this case the power constraint $\bar{\alpha}^{\circ}$ is infeasible, let $y$ denote the minimum $t$ such that $P^{\mathrm{av}}\left(t, t^{\circ}\right)<P^{\mathrm{av}}\left(t, t^{\star}\right)$. Reminding that $\tilde{\phi}_{D}(t-1)+\tilde{F}(t)=\tilde{\phi}_{D}(t)$ in the numerator of (39), we have:

$$
\begin{aligned}
& \tilde{\alpha}^{\circ}(t)=\tilde{\alpha}^{\star}(t) \text { if } t<y \\
& \tilde{\alpha}^{\circ}(y)>\tilde{\alpha}^{\star}(y) \\
& \tilde{\alpha}^{\circ}(t) \geq \tilde{\alpha}^{\star}(t) \text { if } t>y
\end{aligned}
$$

As a result of (C4) and (35), there always exists $\tilde{j} \in \mathscr{N}$ such that $\tilde{\phi}_{\alpha_{\tilde{j}}}^{\circ}(T)>\tilde{\phi}_{\alpha_{\tilde{j}}}^{\star}(T)=t_{j}^{\mathrm{r}}$, thus violating the feasibility condition $(\mathrm{C} 1 \mathrm{~b})$ and concluding the proof.
thus viouting the feasibility condition (C1b) and concluding the proof. 


\section{Tables}

Table C1. Number of macro-appliances $\hat{N}$, computational time $T_{C}$ and demand error $\delta_{C}$ for different values of the clustering parameter $k$.

\begin{tabular}{|c|c|c|c|}
\cline { 2 - 4 } \multicolumn{1}{c|}{} & $\hat{N}$ & $T_{c}(\mathrm{~s})$ & $\delta_{c}(\mathrm{GW})$ \\
\hline$k=4$ & 1006 & 2287.1 & $3.22 \cdot 10^{-4}$ \\
\hline$k=2$ & 566 & 1988.1 & $3.83 \cdot 10^{-4}$ \\
\hline$k=1$ & 340 & 682.2 & $8.23 \cdot 10^{-3}$ \\
\hline$k=0.5$ & 224 & 620.7 & $1.86 \cdot 10^{-2}$ \\
\hline
\end{tabular}




\section{List of Figure Captions}

- Fig. 1: Interactions and information exchange between the different system components.

- Fig. 2: Comparison of a price signal $p$ (top-left) and an arbitrary function $g$ (topright) with the associated quantities $\bar{p}$ and $\bar{g}$ in the reordered time variables (bottom).

- Fig. 3: Top: profiles of inflexible (blue trace), flexible (blue area) and aggregate demand (red trace) when clustered appliances are considered. Bottom: optimal proportional constraint for the clustered optimisation problem (27).

- Fig. 4: Average electricity price paid by the single appliance, as a function of its initial availability instant $t^{\text {in }}$, for different energy ratings $E^{\mathrm{r}}$.

- Fig. 5: Flexible demand $\bar{D}_{f}^{\diamond}$ induced by the clustered population and the corresponding profiles $\bar{D}_{f}$ obtained by broadcasting $\bar{\alpha}^{\diamond}$ (calculated for different values of $k$ ), to the original population.

- Fig.6: Inflexible demand profile $\left(D_{i}\right)$, aggregate demand when the Optimal Scheduling $\left(D_{a}^{O S}\right)$ the Unconstrained Cost Minimisation $\left(D_{a}^{U C M}\right)$ and the Greedy Power Allocation $\left(D_{a}^{G P A}\right)$ are applied.

- Fig. 7: Aggregate demand $\bar{D}_{a}^{\diamond}$ (top) and proportional power constraint $\bar{\alpha}^{\diamond}$ (bottom) for the case study of Section 5. 

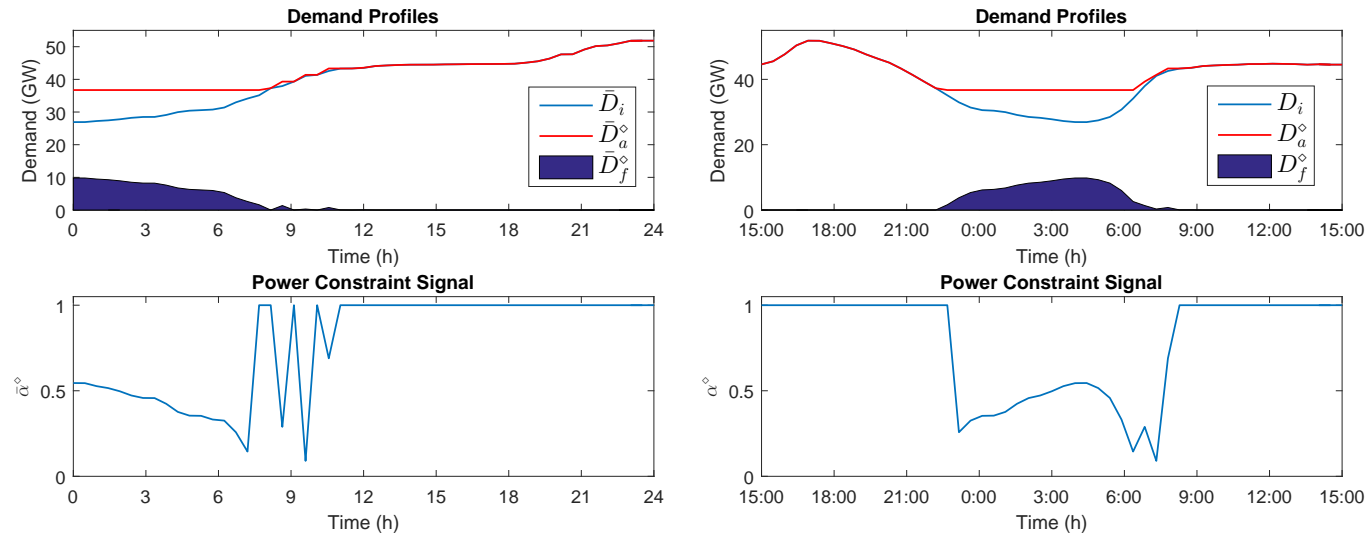

(a) Reordered time vector.

(b) Original time variable.

Figure C3. Top: profiles of inflexible (blue trace), flexible (blue area) and aggregate demand (red trace) when clustered appliances are considered. Bottom: optimal proportional constraint for the clustered optimization problem (27).

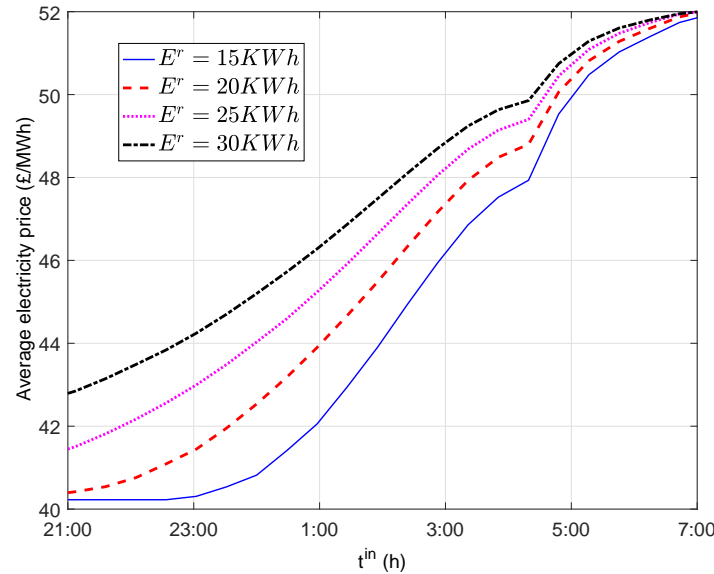

Figure C4. Average electricity price paid by the single appliance, as a function of its initial availability instant $t^{\text {in }}$, for different energy ratings $E^{\mathrm{r}}$.

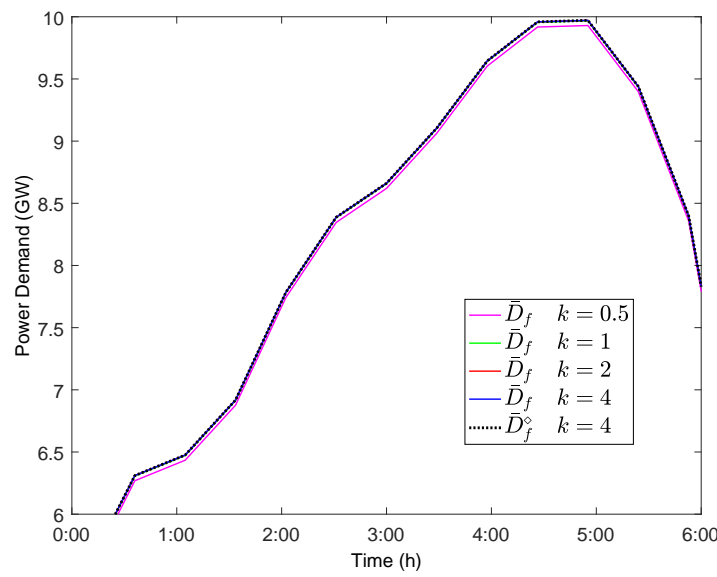

Figure C5. Flexible demand $\bar{D}_{f}^{\diamond}$ induced by the clustered population and the corresponding profiles $\bar{D}_{f}$ obtained by broadcasting $\bar{\alpha}^{\diamond}$ (calculated for different values of $k$ ), to the original population. 


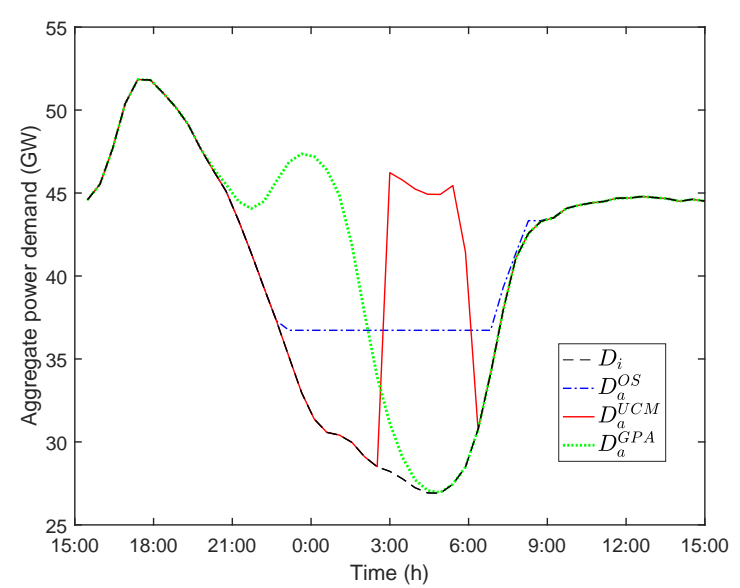

Figure C6. Inflexible demand profile $\left(D_{i}\right)$, aggregate demand when the Optimal Scheduling $\left(D_{a}^{\mathrm{OS}}\right)$ the Unconstrained Cost Minimization $\left(D_{a}^{\mathrm{UCM}}\right)$ and the Greedy Power Allocation $\left(D_{a}^{\mathrm{GPA}}\right)$ are applied.
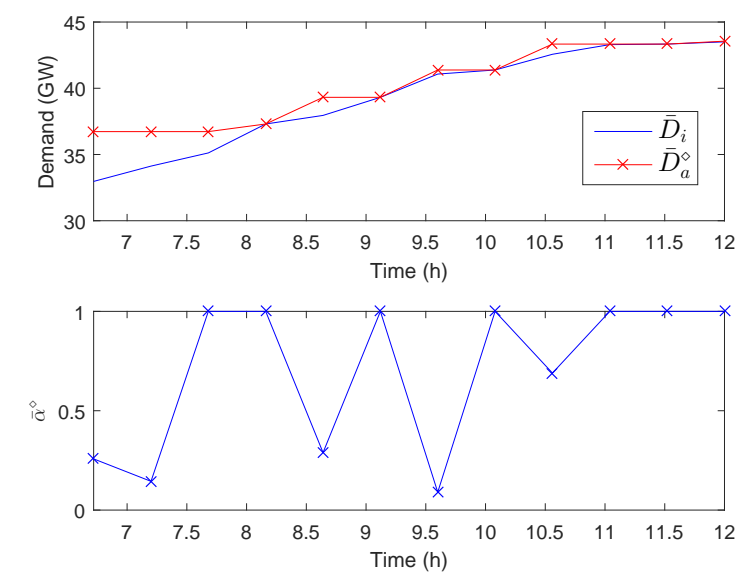

Figure C7. Aggregate demand $\bar{D}_{a}^{\diamond}$ (top) and proportional power constraint $\bar{\alpha}^{\diamond}$ (bottom) for the case study of Section 5 .

the case study of Section 5 